\title{
Lipoma Arborescens: A Rare Extra-Articular Manifestation of Extensor Tendons of the Wrist
}

\author{
Kastanis $\mathrm{G}^{1 *}$, Magarakis $\mathrm{G}^{1}$, Velivasakis $\mathrm{G}^{1}$, Kapsetakis $\mathrm{P}^{1}$, Spyrantis $\mathrm{M}^{1}$ and Pantouvaki $\mathrm{A}^{2}$ \\ ${ }^{1}$ Department of Orthopaedic, General Hospital of Heraklion Venizeleio, Greece \\ ${ }^{2}$ Department of Physiotherapy, Greece
}

*Corresponding author: Grigorios Kastanis, Department of Orthopaedic, General Hospital of Heraklion Venizeleio, Crete, Greece.

Received Date: October 09, 2019

Published Date: October 18, 2019

\begin{abstract}
Lipoma arborescens is a benign tumor of the synovial, which appears, in majority of cases, unilaterally around the knee joint but in rare cases it can also be seen around other large joints (hip, ankle) or tendon sheaths. Extremely rarely, revelation has been reported on wrist or hand. The etiology is unclear and is characterized by the replacement of sub synovial tissue with mature fat cells giving rise to a villous proliferation. We report a 58 years old female with a dorsal palpable soft growing mass on dorsal surface of the wrist in which initially ultrasound diagnosis revealed gaglion cyst, while biopsy after surgical excision determined lipoma arborescens. The aim of this study is to identify that lipoma arborences should be considered in differential diagnosis of soft tumors of the wrist.
\end{abstract}

Keywords: Lipoma arborescens; Soft tissue tumors; Wrist tumors; Tendon sheath; Magnetic resonance imaging

\section{Introduction}

Synovial lipomatosis or Lipoma arborescens (L.A.) is a synovial disorder that usually affects a single joint. The lesion is of unknown etiology and it is unclear whether the condition is postinflammatory, neoplastic or metaplastic [1]. It is a rare benign, intra-articular lesion and is characterized by the replacement of subsynovial tissue with mature fat cells giving rise to a villous proliferation [2]. The most common finding is a slow growing swelling. Usually the condition is being limited to the synovium of a single major joint (knee or hip) but there are some reports claiming that nearby structures can also be affected. The aim of this study is to report a case with an extra-articular dorsal Lipoma arborescens of the right wrist, localization on extensor tendons, which was firstly diagnosed as a gaglion cyst (Ultrasound)and secondly, after surgical excision, was differentially diagnosed as a LA (Biopsy).

\section{Case Report}

A 58 years old female with dorsal carpal pain of the right wrist was proceeded in our clinic. The patient was complaining of a palpable soft growing mass on dorsal surface of the wrist. Patient reported an increase of the mass size the last two months. In clinical examination a soft mass of $7 \times 3,5 \mathrm{~cm}$ was revealed on the dorsal side of the wrist (Figure1). There were no neurological symptoms. Motion of the radiocarpal joint was maintained at normal range, while there was a restriction in extension of digits as a result of mild pain. Radiography (F-Pr-L) of the right wrist had no significant findings. A sonogram and serologic blood tests were ordered. Serologic tests were negative, and the sonogram presented the mass as a gaglion cyst. The patient was undergone excision of the mass. A dorsal incision of $8 \mathrm{~cm}$ was performed on the wrist between 3rd and 4th extensor compartment (Figure 2).

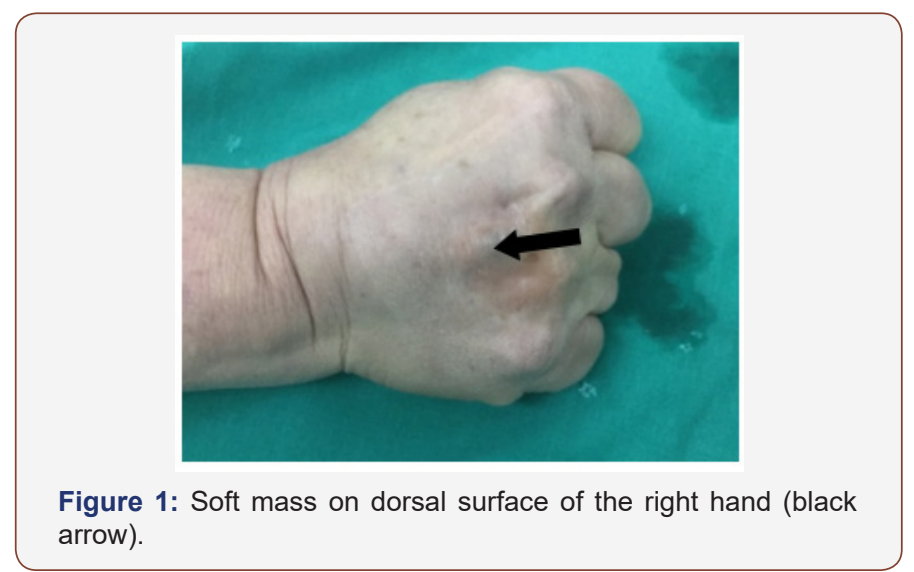




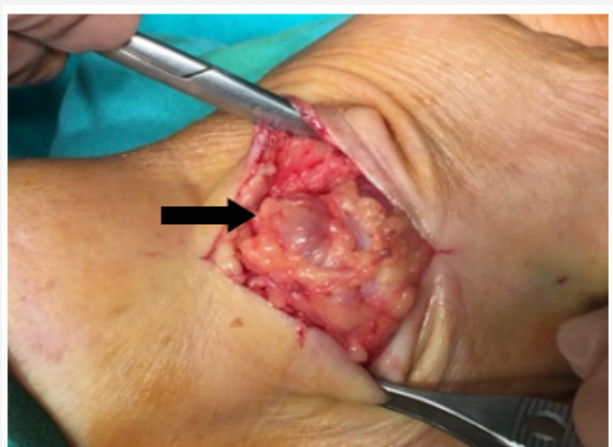

Figure 2: Dorsal aspect of the wrist, the mass has been exposed (black arrow)

A palpable subcutaneous mass was explored. Then synovectomies were further performed and numerousinflammatory villous were identified at the palmar aspect of the mass in a close relation to the extensor tendons in 3rd and 4th compartment (Figure 3). Extensor retinaculum was longitudinally opened over the fourth compartment. There was erosion on the extensor digitorum communes with no further involvement of the rest extensors. The mass was retracted and removed, while the nearby structures were thoroughly cleared (Figure 4). Postoperatively the wrist was immobilized with a half plaster of Paris for two weeks. After that a rehabilitation protocol was followed, to restore full range of motion in all joints having a good functional outcome. The biopsy results of the mass settled the diagnosis of lipoma arborences.

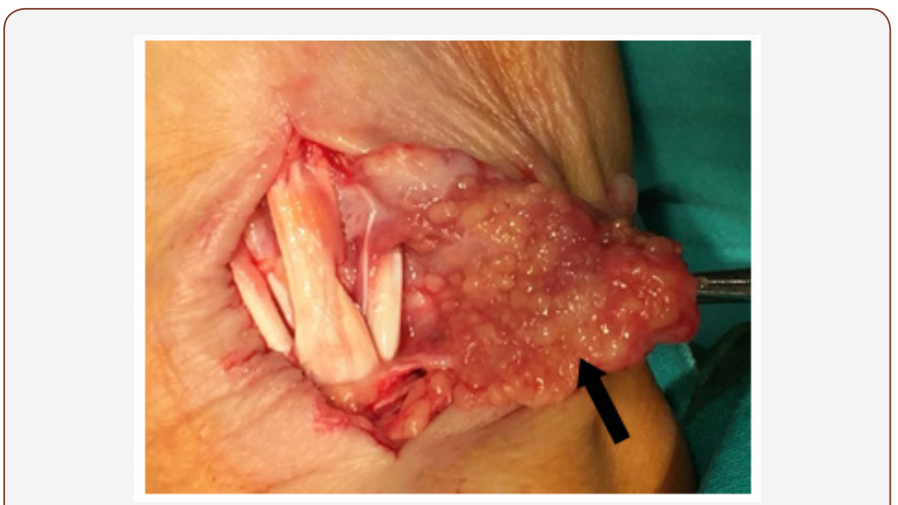

Figure 3: Villous proliferation of the palmar aspect of extensor retinaculum of the wrist (black arrow)

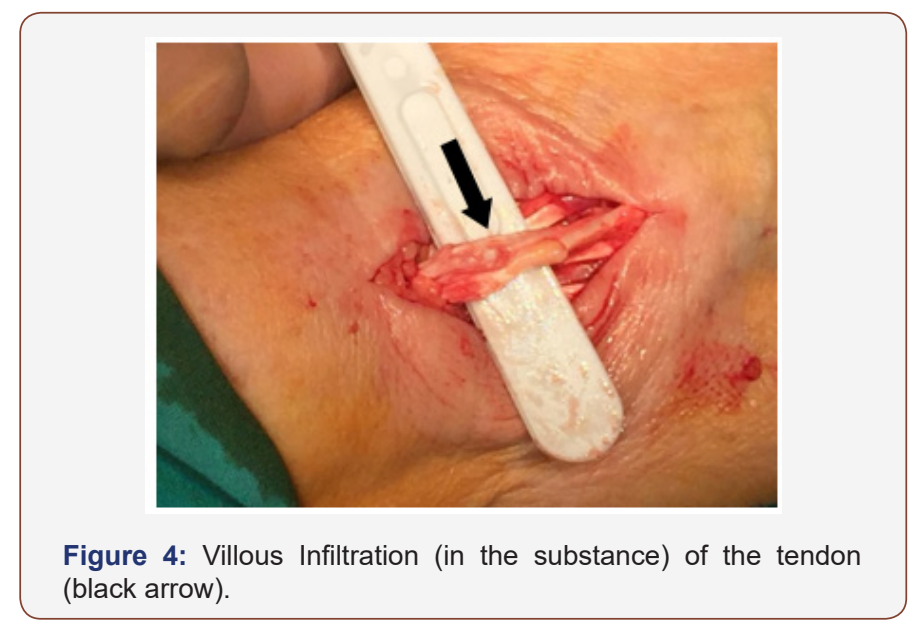

\section{Discussion}

Lipoma Arborescens (L.A.) was first described by Hoffa in 1904, and more in detail in 1957 by Arzimanoglu [3]. The name "arborescens" comes from the Latin word arbor, that means tree, due to the tree like morphology of lipomatous villi proliferation. It is considered like a villous synovial hyperplastic process rather than a real neoplasm [1]. The etiology is unknown but is associated with local trauma, chronic Degenerative arthritis, Rheumatoid arthritis, Diabetes Mellitus or steroids use [4,5]. It is more frequent in middle aged men (40-60 years old). Most of these lesions are met in large single joints and the localization is intra -articular. It is limited in the synovium without invasion of the other structures. The clinical manifestation is characterized by joint pain, clicking, effusion or locking due to interposition of villous between join surface [6].

Knee joint is the most commonly affected, mainly in the suprapatellar pouch [5]. Cases involving other locations including shoulder, hip, elbow and ankle have been rarely described [7]. Santiago et al. (2009) describe a 29-year-old female with polyarticular lesion (wrists, knees, ankles) mimicking rheumatoid arthritis, while Al- Ismail presents a case of bilateral knee lesion with associated marked premature osteoarthritis [3]. Siva et al report a case with multifocal involvement (hands, wrists, knees) with congenital short bowel syndrome [8]. It is suggested that the predisposing condition of the LA is the abnormal retention of preadipocytes or multi/pluripotent stem cells in the sub synovial region [3]. Extra-articular localization of L.A. is rarely reported. Huang et al described a case of a 12-year-old girl with lipoma arborescence, affecting the synovial sheaths of the peroneal, posterior tibialis, and flexor tendons. Localization of lipoma arborescens of the extensor tendons of the wrist and hand is extremely rare and only a few cases have been mentioned. Chandler et al noted a rare manifestation of L.A. in bilateral wrist, while Kamaci et al. report a case of L.A secondary to foreign body reaction, in the extensor tendon sheath after an industrial accident. Hill and Phyto (2011) describe a case with L.A. on the dorsal surface on the hand with a scaphoid fracture and degenerative radio-scaphoid arthritis. Stepan et al (2013) report a case of extra- articular L.A. with direct invasions of fourth extensor compartment of the wrist with erosion of almost the $50 \%$ of the tendon's substance of the index and long finger1. In our case the lesion is extra-articular localized under the extensor tendon sheath of the 3rd and 4th compartment with villous proliferation in tendon substance. The patient had a painful slow growing mass in the dorsum of the wrist, with no previous history of trauma and without irritation of the joint as in inflammatory disease.

Magnetic resonance imaging (MRI) is the test of choice for the diagnosis of the lesion. Bernando et al describe that MRI turns out the preoperative biopsy unnecessary [5]. The MRI shows fatty synovial proliferation with no other signal intensities and this is the pathognomonic criterion for the diagnosis of lipoma arborescens. The presence of fat is confirmed at T1 weight images; the high signal intensity of the fat becomes darker on a STIR. The plain radiography, 
ultrasound imaging and laboratory tests are non-specific but are used to exclude other pathologies [5,9]. The differential diagnosis accounts to synovial lipoma, synovial chondromatosis, pigmented villonodular synovitis, synovial haemangioma, and rheumatoid arthritis [10].

Conservative treatment is indicated for patients with such lesions with small dimensions, that cause no pain or functional limitations, otherwise surgical resection is recommended [11]. The choice of the technique (open or arthroscopic) depends on the size of the mass the location of the lesion (intra-extraarticular), and the preference of the surgeon [12]. We postulated that when localization is intra-articular arthroscopy excision offers good outcomes, while when lesion is extraarticular the open rejection is the technique of choice [13].

\section{Conclusion}

Extra-articular lipoma arborescens of extensor tendon of the wrist is a rare lesion. Magnetic resonance imaging has the essential role in the diagnosis of the lesion and in the differentiation from other pathologies. The treatment (conservative-surgical) depends on the characteristics of the mass (size, localization, mono-polyarticular) and on functional status of the patient (pain, restriction of motion), while when surgical treatment is mandatory the technique chosen depends on the type of lesion.

\section{Ethics Approval}

Our institution does not require ethical approval for reporting individual cases or case series.

\section{Informed Consent}

Verbal informed consent was obtained from the patient(s) for their anonymized information to be published in this article.

\section{Acknowledgement}

None.

\section{Conflict of Interest}

The authors declare that there is no conflict of interest. We certify that no funding has been received for the conduct of this study and/or preparation of this text.

\section{References}

1. Stepan JG, Gelberman RH, Rubin DA, Osei DA (2013) Extra-Articular Lipoma Arborescens of the Dorsal Aspect of the Wrist with Invasion of the Extensor Tendons: A Case Report. JBJS Case Connect 3(1): e30.

2. Bejia I, Younes M, Moussa A, Said M, Touzi M, et al. (2005) Lipoma arborescens affecting multiple joints. Skeletal Radiol 34(9): 536-538.

3. De Vleeschhouwer M, Van Den Steen E, Vanderstraeten G, Huysse W, De Neve J, et al. (2016) Lipoma Arborescens: Review of an Uncommon Cause for Swelling of the Knee. Case Reports in Orthopedics.

4. Hallel T, Lew S, Bansal M (1988) Villous lipomatous proliferation of the synovial membrane (lipoma arborescens). J Bone Joint Surg Am 70(2): 264-270.

5. Vilanova JC, Barcelo J, Villalon M, Aldoma J, Delgado E, et al. (2003) MR imaging of lipoma arborescens and the associated lesions. Skeletal Radiol 32 (9): 504-509.

6. Bernardo A, Bernardes M, Brito I, Vieira A, Ventura F (2004) Lipoma arborescente da synovial. Acta Med Port 17: 325-328.

7. Siva C, Brasington R, Totty W, Sotelo A, Atkinson J (2002) Synovial lipomatosis (lipoma arborescens) affecting multiple joints in a patient with congenital short bowel syndrome. J Rheumatol 29(5): 1088-1092.

8. Tsifountoudis I, Kapoutsis D, Tzavellas AN, Kalaitziglou I, Tsikes A, et al. (2017) Lipoma arborescens of the knee: Report of three cases and review of the literature. Case Rep Med 3569512.

9. Grieten M, Buckwalter KA, Cardinal E, Rougraff B (1994) Case report 873: lipoma arborescens (villous lipomatous proliferation of the synovial membrane. Skeletal Radiol 23(8): 652-655.

10. Kamaci S, Ergen FB, Leblebicioglu G (2015) Lipoma arborescens in the extensor tendon sheath of the hand secondary to foreign body reaction. J Hand Surg Eur Vol 40(7): 751-753.

11. Huang GS, Lee HS, Hsu YC, Kao HW, Lee HH, et al. (2006) Tenosynovial lipoma arborescens of the ankle in a child. Skeletal Radiol 35(4): 244247.

12. Chander B, Awasthi B, Preet K (2015) Synchronous Lipoma arborescens of bilateral wrist: An extremely rare manifestation and a new perspective on etiopathogenesis. J Cancer Res Ther 11(3): 646.

13. Hill GN, Pyho N (2011) Extra-articular lipoma arborescens of the hand: An unusual case report. J Hand Surg (Eur) 36(5): 422-423. 